\title{
THE MULTIPLICITY CONJECTURE IN LOW CODIMENSIONS
}

\author{
Juan Migliore, Uwe Nagel, and Tim Römer
}

\begin{abstract}
We establish the multiplicity conjecture of Herzog, Huneke, and Srinivasan about the multiplicity of graded Cohen-Macaulay algebras over a field, for codimension two algebras and for Gorenstein algebras of codimension three. In fact, we prove stronger bounds than the conjectured ones allowing us to characterize the extremal cases. This may be seen as a converse to the multiplicity formula of Huneke and Miller that inspired the conjectural bounds.
\end{abstract}

\section{Introduction}

Let $R=K\left[x_{1}, \ldots, x_{n}\right]$ be the polynomial ring over a field $K$ with its standard grading where $\operatorname{deg} x_{i}=1$ for $i=1, \ldots, n$. Let $R / I$ be a standard graded $K$ algebra, where $I$ is a graded ideal of codimension $c$. We denote by $e(R / I)$ the multiplicity of $R / I$. When $I$ is a saturated ideal defining a closed subscheme $V \subset \mathbb{P}^{n-1}, e(R / I)$ is just the degree $\operatorname{deg} V$ of $V$. Consider the minimal graded free resolution of $R / I$

$$
0 \rightarrow \bigoplus_{j \in \mathbb{Z}} R(-j)^{\beta_{p, j}^{R}(R / I)} \rightarrow \cdots \rightarrow \bigoplus_{j \in \mathbb{Z}} R(-j)^{\beta_{1, j}^{R}(R / I)} \rightarrow R \rightarrow R / I \rightarrow 0
$$

where we denote by $\beta_{i, j}^{R}(R / I)=\operatorname{Tor}_{i}^{R}(R / I, K)_{j}$ the graded Betti numbers of $R / I$ and $p$ is the projective dimension of $R / I$. Let $c$ denote the codimension of $R / I$. Then $c \leq p$ and equality holds if and only if $R$ is Cohen-Macaulay. We define $m_{i}=\min \left\{j \in \mathbb{Z}: \beta_{i, j}^{R}(R / I) \neq 0\right\}$ and $M_{i}=\max \left\{j \in \mathbb{Z}: \beta_{i, j}^{R}(R / I) \neq 0\right\}$. When there is any danger of ambiguity, we write $m_{i}(I)$ and $M_{i}(I)$. The algebra $R / I$ has a pure resolution if $m_{i}=M_{i}$ for all $i$. In this case we write $d_{i}$ for the unique $j$ such that $\beta_{i, j}^{R}(R / I) \neq 0$. It was shown by Huneke and Miller [13] that if $R / I$ is Cohen-Macaulay with a pure resolution then

$$
e(R / I)=\left(\prod_{i=1}^{p} d_{i}\right) / p !
$$

Extending this, Herzog, Huneke and Srinivasan made the following multiplicity conjecture:

Received by the editors October 22, 2004.

Part of the work for this paper was done while the first author was sponsored by the National Security Agency under Grant Number MDA904-03-1-0071 and the second author was supported by a Special Faculty Research Fellowship from the University of Kentucky. 
Conjecture 1.1. If $R / I$ is Cohen-Macaulay then

$$
\left(\prod_{i=1}^{p} m_{i}\right) / p ! \leq e(R / I) \leq\left(\prod_{i=1}^{p} M_{i}\right) / p ! .
$$

Conjecture 1.1 has been studied extensively, and partial results have been obtained. In [11], Herzog and Srinivasan proved it in the following cases: $R / I$ is a complete intersection; $I$ is a perfect ideal with quasi-pure resolution (i.e. $\left.m_{i}(R / I) \geq M_{i-1}(R / I)\right) ; I$ is a perfect ideal of codimension $2 ; I$ is a codimension 3 Gorenstein ideal with five minimal generators; $I$ is a Gorenstein monomial ideal of codimension 3 with at least one generator of smallest possible degree (relative to the number of generators); $I$ is a perfect stable ideal; $I$ is a perfect squarefree strongly stable ideal. Furthermore, Herzog and Srinivasan proved that the upper bound of Conjecture 1.1 holds for all codimension 3 Gorenstein ideals. Guardo and Van Tuyl [10] proved that the conjecture holds for powers of complete intersections, and Gold, Schenck and Srinivasan [8] proved it in certain cases where $I$ is linked to something "simple." In addition, Srinivasan [20] proved a stronger bound for Gorenstein algebras with quasi-pure resolutions. (cf. Remark 3.3.)

The non Cohen-Macaulay case has also been studied. Here it is necessary to replace the projective dimension by the codimension in Conjecture 1.1. It was observed in [11] that the lower bound is false. However, Herzog and Srinivasan proved the upper bound in these cases: $I$ is a stable ideal; $I$ is a squarefree strongly stable ideal; $I$ is an ideal with $d$-linear resolution. In addition, Gold [7] proved it for codimension two lattice ideals; this was generalized by Römer [18] for all codimension two ideals. It was also proved by Gasharov, Hibi and Peeva [4] for a-stable ideals and more generally by Römer [18] for componentwise linear ideals.

In this paper we begin with a new, stronger version of Conjecture 1.1 in the codimension two case:

Theorem 1.2. Let $R / I$ be a graded Cohen-Macaulay algebra of codimension two. Then the following lower and upper bounds hold:

(a) $e(R / I) \geq \frac{1}{2} m_{1} m_{2}+\frac{1}{2}\left(M_{2}-M_{1}\right)\left(M_{2}-m_{2}+M_{1}-m_{1}\right)$;

(b) $e(R / I) \leq \frac{1}{2} M_{1} M_{2}-\frac{1}{2}\left(m_{2}-m_{1}\right)\left(M_{2}-m_{2}+M_{1}-m_{1}\right)$.

As an immediate consequence of this result, we obtain the following characterization for the sharpness of Conjecture 1.1 in the codimension two CohenMacaulay case. This can be viewed as a converse to the Huneke-Miller result [13] mentioned above.

Corollary 1.3. Let $R / I$ be a graded Cohen-Macaulay algebra of codimension two. Then the following conditions are equivalent:

(a) $e(R / I)=\frac{1}{2} m_{1} m_{2}$;

(b) $e(R / I)=\frac{1}{2} M_{1} M_{2}$;

(c) $R / I$ has a pure minimal graded free resolution. 
In Section 2 we give two proofs of these results. The first is based on some formulas in [11]. The second one is more self-contained and uses the methods that allow us obtain the results discussed below. We also discuss the non CohenMacaulay case. In particular, we show that even a natural weakening of the lower bound in Conjecture 1.1 could only be true for reduced ideals (cf. Remark 2.4). In Section 3 we prove a stronger version of Conjecture 1.1 for Gorenstein ideals of codimension three:

Theorem 1.4. Let $R / I$ be a graded Gorenstein algebra of codimension three. Then the following lower and upper bounds hold:

(a) $e(R / I) \geq \frac{1}{6} m_{1} m_{2} m_{3}+\frac{1}{6}\left(M_{3}-M_{2}\right)^{2}\left(M_{2}-m_{2}+M_{1}-m_{1}\right)$;

(b) $e(R / I) \leq \frac{1}{6} M_{1} M_{2} M_{3}-\frac{1}{12} M_{3}\left(M_{2}-m_{2}+M_{1}-m_{1}\right)$.

As an immediate consequence we get that the lower bound of Conjecture 1.1 holds for codimension three Gorenstein ideals. This was the last open case of the conjecture in low codimensions where structure theorems of minimal graded free resolutions are available. As in the case of codimension two perfect ideals we can characterize when Conjecture 1.1 is sharp providing again a converse to the Huneke-Miller formula in [13].

Corollary 1.5. Let $R / I$ be a graded Gorenstein algebra of codimension three. Then the following conditions are equivalent:

(a) $e(R / I)=\frac{1}{6} m_{1} m_{2} m_{3}$;

(b) $e(R / I)=\frac{1}{6} M_{1} M_{2} M_{3}$;

(c) $R / I$ has a pure minimal free resolution.

Our method of proof consists in exhibiting a specific example for each possible set of Betti numbers and a reduction procedure that allows us to proceed by induction. While the first idea seems difficult to extend we expect that the reductions via basic double links will be useful in other cases, too.

We conclude this note with an explicit formula for the multiplicity of a Gorenstein ideal in terms of the degrees of the entries of its Buchsbaum-Eisenbud matrix (Proposition 3.4). It is a by-product of the proof of Theorem 1.4.

\section{Codimension two Cohen-Macaulay algebras}

Let $K$ be a field, $R=K\left[x_{1}, \ldots, x_{n}\right]$ be the polynomial ring and $I \subset R$ a graded ideal of height two such that $R / I$ is a Cohen-Macaulay ring. It follows from the Hilbert-Burch theorem (e.g. see [1] for details) that $I$ has a minimal graded free resolution of the form

$$
0 \rightarrow \bigoplus_{i=1}^{m-1} R\left(-f_{i}\right) \stackrel{\varphi}{\longrightarrow} \bigoplus_{i=1}^{m} R\left(-e_{i}\right) \rightarrow I \rightarrow 0
$$

Let $u_{i}=f_{i}-e_{i}$ and $v_{i}=f_{i}-e_{i+1}$. The following was observed, for example, in [12]:

(a) $u_{i} \geq v_{i} \geq 0$ for $i=1, \ldots, m-1$; 
(b) $u_{i+1} \geq v_{i}$ for $i=1, \ldots, m-2$;

(c) $e_{1}=v_{1}+\cdots+v_{m-1}$ and $e_{m}=u_{1}+\cdots+u_{m-1}$;

(d) $f_{1}=v_{1}+\cdots+v_{m-1}+u_{1}$ and $f_{m-1}=u_{1}+\cdots+u_{m-1}+v_{m-1}$;

(e) $e(R / I)=\sum_{i=1}^{m-1} u_{i}\left(v_{i}+\cdots+v_{m-1}\right)=\sum_{i=1}^{m-1} v_{i}\left(u_{1}+\cdots+u_{i}\right)$.

Herzog and Srinivasan proved in [11] the formulas (see proof of Theorem 2.1):

(a) $\sum_{i=2}^{m-1}\left(v_{i-1}+v_{i}\right)\left(v_{i}+\cdots+v_{m-1}\right)=\left(v_{1}+\cdots+v_{m-1}\right)\left(v_{2}+\cdots+v_{m-1}\right)$;

(b) $\sum_{i=1}^{m-2}\left(u_{i}+u_{i+1}\right)\left(u_{1}+\cdots+u_{i}\right)=\left(u_{1}+\cdots+u_{m-1}\right)\left(u_{1}+\cdots+u_{m-2}\right)$.

Note that $e_{1}=m_{1}, e_{m}=M_{1}, f_{1}=m_{2}$ and $f_{m-1}=M_{2}$. Following the proof of Theorem 2.1 in [11], we can show Theorem 1.2 of the introduction.

Proof of Theorem 1.2. (a) Using

$$
\begin{array}{r}
2 u_{1}=\left(u_{1}-v_{1}\right)+u_{1}+v_{1}=e_{2}-e_{1}+u_{1}+v_{1} \\
2 u_{i}=\left(2 u_{i}-v_{i-1}-v_{i}\right)+v_{i-1}+v_{i}=\left(e_{i+1}-e_{i}+f_{i}-f_{i-1}\right)+v_{i-1}+v_{i}
\end{array}
$$

and the first formula of Herzog and Srinivasan above we compute

$$
\begin{aligned}
2 e(R / i)= & \sum_{i=1}^{m-1} 2 u_{i}\left(v_{i}+\cdots+v_{m-1}\right) \\
= & e_{1} f_{1}+\left(u_{1}-v_{1}\right)\left(v_{1}+\cdots+v_{m-1}\right) \\
& +\sum_{i=2}^{m-1}\left(2 u_{i}-v_{i}-v_{i-1}\right)\left(v_{i}+\cdots+v_{m-1}\right) \\
= & m_{1} m_{2}+\left(e_{2}-e_{1}\right)\left(v_{1}+\cdots+v_{m-1}\right)+ \\
& \sum_{i=2}^{m-1}\left(e_{i+1}-e_{i}+f_{i}-f_{i-1}\right)\left(v_{i}+\cdots+v_{m-1}\right) \\
= & m_{1} m_{2}+\sum_{i=1}^{m-1}\left(e_{i+1}-e_{i}\right)\left(v_{i}+\cdots+v_{m-1}\right) \\
& +\sum_{i=2}^{m-1}\left(f_{i}-f_{i-1}\right)\left(v_{i}+\cdots+v_{m-1}\right) \\
\geq & m_{1} m_{2}+v_{m-1}\left(\sum_{i=1}^{m-1}\left(e_{i+1}-e_{i}\right)+\sum_{i=2}^{m-1}\left(f_{i}-f_{i-1}\right)\right) \\
= & m_{1} m_{2}+\left(M_{2}-M_{1}\right)\left(M_{1}-m_{1}+M_{2}-m_{2}\right) .
\end{aligned}
$$

Dividing by 2 , the desired formula follows. 
(b) Similarly, using the second formula of Herzog and Srinivasan we compute

$$
\begin{aligned}
2 e(R / I)= & \sum_{i=1}^{m-1} 2 v_{i}\left(u_{1}+\cdots+u_{i}\right) \\
= & e_{m} f_{m-1}+\left(v_{m-1}-u_{m-1}\right)\left(u_{1}+\cdots+u_{m-1}\right) \\
& +\sum_{i=1}^{m-2}\left(2 v_{i}-u_{i}-u_{i+1}\right)\left(u_{1}+\cdots+u_{i}\right) \\
= & M_{1} M_{2}-\left(e_{m}-e_{m-1}\right)\left(u_{1}+\cdots+u_{m-1}\right) \\
& -\sum_{i=1}^{m-2}\left(e_{i+1}-e_{i}+f_{i+1}-f_{i}\right)\left(u_{1}+\cdots+u_{i}\right) \\
= & M_{1} M_{2} \\
& -\sum_{i=1}^{m-1}\left(e_{i+1}-e_{i}\right)\left(u_{1}+\cdots+u_{i}\right)-\sum_{i=1}^{m-2}\left(f_{i+1}-f_{i}\right)\left(u_{1}+\cdots+u_{i}\right) \\
\leq & M_{1} M_{2}-u_{1}\left(\sum_{i=1}^{m-1}\left(e_{i+1}-e_{i}\right)+\sum_{i=1}^{m-2}\left(f_{i+1}-f_{i}\right)\right) \\
= & M_{1} M_{2}-\left(m_{2}-m_{1}\right)\left(M_{1}-m_{1}+M_{2}-m_{2}\right)
\end{aligned}
$$

We have found a second proof for both parts of Theorem 1.2, using some methods of liaison theory. This proof has the advantage that it is more selfcontained, and also that it is very much in the spirit of the (more complicated) proof for codimension three Gorenstein ideals given in the next section, which incorporates the ideas from this proof and proceeds from there. On the other hand, since Theorem 1.2 is now proven, we will only give part of the alternative proof, and set up the machinery that is needed for the next section.

The map $\varphi$ in the minimal free resolution (1) is represented by the HilbertBurch matrix of $I$. By reordering we can arrange that the degree matrix is

$$
A=\left[\begin{array}{cccc}
a_{1,1} & a_{1,2} & \ldots & a_{1, t+1} \\
\vdots & \vdots & & \vdots \\
a_{t, 1} & a_{t, 2} & \ldots & a_{t, t+1}
\end{array}\right]
$$

where the entries are increasing from bottom to top and from left to right, so $a_{t, 1}$ is the smallest and $a_{1, t+1}$ is the largest. Notice that in order to be a degree matrix of a height two Cohen-Macaulay ideal, the main diagonal has to be strictly positive ([5], page 3142 and see [19], page 84).

Remark 2.1. Let us rewrite the degree matrix $A$ as follows

$$
A=\left[\begin{array}{cccc}
a_{1} & b_{1} & & * \\
& \ddots & \ddots & \\
* & & a_{t} & b_{t}
\end{array}\right]
$$


Note that $A$ is completely determined by $a_{1}, \ldots, a_{t}, b_{1}, \ldots, b_{t}$. By our ordering of degrees we have in particular

$$
b_{t} \geq a_{t} \quad \text { and } \quad b_{t-1} \geq a_{t} \text { provided } t \geq 2 .
$$

The minimal generators of $I$ have degrees $a_{1}+\ldots+a_{j}+b_{j+1}+\ldots+b_{t}, j=0, \ldots, t$ and the syzygies of $I$ have degrees $a_{1}+\ldots+a_{j}+b_{j}+\ldots+b_{t}, j=1, \ldots, t$. Thus, we obtain

$$
\begin{aligned}
m_{1} & =a_{1}+\ldots+a_{t} \\
M_{1} & =b_{1}+\ldots+b_{t} \\
m_{2} & =a_{1}+\ldots+a_{t}+b_{t}=m_{1}+b_{t} \\
M_{2} & =a_{1}+b_{1}+\ldots+b_{t} .
\end{aligned}
$$

Alternative proof of Theorem 1.2. We will give most of the details for (a), and leave (b) entirely to the reader. We induct on $t \geq 1$. If $t=1$ then $I$ is a complete intersection, and it is not difficult to prove the result in this case.

Now assume $t \geq 1$ and let $I^{\prime}$ be an ideal whose degree matrix of the HilbertBurch matrix is

$$
A^{\prime}=\left[\begin{array}{ccccc}
a_{1} & b_{1} & & & * \\
& \ddots & \ddots & & \\
& & a_{t} & b_{t} & \\
* & & & a_{t+1} & b_{t+1}
\end{array}\right] .
$$

Note that the multiplicity of $R / I^{\prime}$ is completely determined by the degree matrix, so it suffices to consider an example of an ideal for any degree matrix. Basic double linkage is then used in order to apply the induction hypothesis. The idea will be to show that we can reduce to an ideal with degree matrix $A$ (see Remark 2.1 ), i.e. we remove the last row and the last column.

It is easy to see that the following monomial ideal has $A^{\prime}$ as its degree matrix

$$
I^{\prime}=\left(y^{b_{1}+\ldots+b_{t+1}}, x^{a_{1}} y^{b_{2}+\ldots+b_{t+1}}, \ldots, x^{a_{1}+\ldots+a_{t+1}}\right) .
$$

Write it as

$$
I^{\prime}=x^{a_{1}+\ldots+a_{t+1}} R+y^{b_{t+1}} I .
$$

Then the monomial ideal $I$ has $A$ as its degree matrix where $A$ is obtained by deleting the last row and column of $B$. Thus, we may apply induction to $I$. Let $m_{1}, m_{2}, M_{1}, M_{2}$ be the corresponding invariants for $I$, and let $m_{1}^{\prime}, m_{2}^{\prime}, M_{1}^{\prime}, M_{2}^{\prime}$ be those of $I^{\prime}$. Moreover, in order to simplify notation we set

$$
a:=a_{t+1}, b:=b_{t+1}, \text { and } c:=b_{t} .
$$

Using Remark 2.1, we see that

$$
\begin{aligned}
& m_{1}^{\prime}=m_{1}+a \\
& M_{1}^{\prime}=M_{1}+b \\
& m_{2}^{\prime}=m_{2}+a+b-c \\
& M_{2}^{\prime}=M_{2}+b
\end{aligned}
$$


Moreover, we have $e\left(R / I^{\prime}\right)=e(R / I)+m_{1}^{\prime} b$. Using the formulas above we get by induction

$$
\begin{aligned}
m_{1}^{\prime} m_{2}^{\prime} & +\left(M_{2}^{\prime}-M_{1}^{\prime}\right)\left(M_{2}^{\prime}-m_{2}^{\prime}+M_{1}^{\prime}-m_{1}^{\prime}\right) \\
= & \left(m_{1}+a\right)\left(m_{2}+a+b-c\right) \\
& +\left[\left(M_{2}+b\right)-\left(M_{1}+b\right)\right]\left[\left(M_{2}+b\right)-\left(m_{2}+a+b-c\right)+\left(M_{1}+b\right)-\left(m_{1}+a\right)\right] \\
= & m_{1} m_{2}+\left(m_{1}+a\right)(a+b-c)+m_{2} a \\
& +\left(M_{2}-M_{1}\right)\left[M_{2}-m_{2}+M_{1}-m_{1}+b+c-2 a\right] \\
\leq & 2 e(R / I)+\left(m_{1}+a\right)(a+b-c)+m_{2} a+\left(M_{2}-M_{1}\right)(b+c-2 a) \\
= & 2 e\left(R / I^{\prime}\right)-2\left(m_{1}+a\right) b+\left(m_{1}+a\right)(a+b-c)+\left(m_{1}+c\right) a+ \\
& \left(M_{2}-M_{1}\right)(b+c-2 a) \\
= & 2 e\left(R / I^{\prime}\right)+\left[m_{1}+M_{1}-M_{2}\right](2 a-b-c)+a(a-b) .
\end{aligned}
$$

Note that we used the relation $m_{2}=m_{1}+c$. But $b \geq a$ and $c \geq a$ and $M_{1}+m_{1} \geq M_{2}$ by 2.1 , so

$$
\frac{1}{2} m_{1}^{\prime} m_{2}^{\prime}+\frac{1}{2}\left(M_{2}^{\prime}-M_{1}^{\prime}\right)\left(M_{2}^{\prime}-m_{2}^{\prime}+M_{1}^{\prime}-m_{1}^{\prime}\right) \leq e\left(R / I^{\prime}\right)
$$

as desired. (Note the strict inequality unless $a=b=c$ or $t=2$ and $a=b$.)

The upper bound in Theorem 1.2(b) is proved in a similar way, and we omit the details.

Using this approach, we can prove another upper bound that extends the upper bound of Herzog and Srinivasan. The following proposition has a hypothesis that is a bit technical, but it has a more satisfying conclusion than the bound of Theorem 1.2(b) in one case.

Proposition 2.2. Let I be a height two Cohen-Macaulay ideal with degree matrix

$$
A=\left[\begin{array}{ccccc}
a_{1} & b_{1} & & & * \\
d & a_{2} & b_{2} & & \\
& & \ddots & \ddots & \\
* & & & a_{t} & b_{t}
\end{array}\right]
$$

as in Remark 2.1 (but note the new variable $d$ in the $(1,2)$ spot if $t \geq 2$ ). Then either one of the following conditions is sufficient to conclude that

$$
e(R / I) \leq \frac{1}{2} M_{1} M_{2}-\left(M_{1}-m_{1}\right)-\left(M_{2}-m_{2}\right) .
$$

(i) all of the entries of $A$ are $\geq 2$;

(ii) $a_{1}-2 d+1 \geq 0$, provided $t \geq 2$.

Proof. We omit the details and leave the proof to the reader.

Example 2.3. Consider the degree matrix

$$
B=\left[\begin{array}{llll}
2 & 2 & 2 & 2 \\
2 & 2 & 2 & 2 \\
1 & 1 & 1 & 1
\end{array}\right]
$$


This comes, for example, from the ideal $I^{\prime}=\left(x^{5}, x^{4} y, x^{2} y^{3}, y^{5}\right)$. It is easy to check that $M_{1}^{\prime}=m_{1}^{\prime}=5, M_{2}^{\prime}=7, m_{2}^{\prime}=6$, and $e\left(R / I^{\prime}\right)=17$. If we consider the $2 \times 3$ submatrix $A$, as in the proof, we have $m_{1}=M_{1}=4, m_{2}=M_{2}=6$. Then

$$
e\left(R / I^{\prime}\right)>\frac{1}{2} M_{1}^{\prime} M_{2}^{\prime}-\left(M_{1}^{\prime}-m_{1}^{\prime}\right)-\left(M_{2}^{\prime}-m_{2}^{\prime}\right)=16.5 .
$$

So we see that the conclusion of Proposition 2.2 does not hold here, and indeed $a_{1}-2 d+1=-1$.

In fact, it is not too difficult to show that a $t \times(t+1)$ degree matrix consisting of 2's in the first $(t-1)$ rows and 1's in the last row satisfies $m_{1}=M_{1}=2 t-1$, $m_{2}=2 t, M_{2}=2 t+1$, and $e\left(R / I^{\prime}\right)=2 t^{2}-1$. However, one checks that

$$
\frac{1}{2} M_{2} M_{2}-\left(M_{1}-m_{1}\right)-\left(M_{2}-m_{2}\right)=2 t^{2}-\frac{3}{2}
$$

so this gives an example of any size that violates the bound of Proposition 2.2.

As mentioned in the introduction, it was shown by Huneke and Miller [13] that if $R / I$ is Cohen-Macaulay of codimension $c$ with a pure resolution then

$$
e(R / I)=\left(\prod_{i=1}^{c} d_{i}\right) / c !
$$

where of course $d_{i}=m_{i}=M_{i}$ is the shift in the $i$-th free module of the pure resolution. Corollary 1.3 in the introduction may be seen as a converse to this result.

Proof of Corollary 1.3. The claim follows from Theorem 1.2 because $m_{2}>m_{1}$ and $M_{2}>M_{1}$.

We end this section with a remark about the non Cohen-Macaulay case in codimension two.

Remark 2.4. It is known that the lower bound of Conjecture 1.1 is false in the non Cohen-Macaulay case. Even the weaker statement

$$
e(R / I) \geq \frac{1}{c !} \prod_{i=1}^{c} m_{i}
$$

(where $c$ is the codimension of $I$ ) is false. An easy example is the case of two skew lines in $\mathbb{P}^{3}$. In codimension two, however, the analogous upper bound is true $([18])$. Is there a different lower bound that is true? One natural guess is that one might be able to replace $c$ ! by some suitable integer $k$. That is, perhaps

$$
e(R / I) \geq \frac{1}{k} \prod_{i=1}^{c} m_{i}
$$

for suitable $k$. One immediately sees that at the very least, we should assume that our ideals are unmixed. For instance, starting with any curve, adding points does not change the multiplicity but makes the $m_{i}$ arbitrarily large. 
The next observation is that we must assume that $I$ is reduced in order to hope for a lower bound of the type $\frac{1}{k} \prod_{i=1}^{c} m_{i}$. Indeed, consider ideals in $k\left[x_{0}, x_{1}, x_{2}, x_{3}\right]$ of the form

$$
I=\left(x_{0}, x_{1}\right)^{t}+(F)
$$

where $F$ is a polynomial that is smooth along the line defined by $\left(x_{0}, x_{1}\right)$, and $\operatorname{deg} F \geq t+1$. Then $I$ defines an unmixed curve of multiplicity $t$ (cf. [16]), and one quickly sees that $m_{1}=t$ and $m_{2}=t+1$. Hence we would need $k \geq t+1$, which can be made arbitrarily large by choosing large $t$.

However, if we do assume that $I$ is reduced, there may be such a bound. Indeed, experiments with Macaulay ([9]) have not yet yielded a counterexample to the guess

$$
e(R / I) \geq \frac{1}{5} m_{1} m_{2}
$$

at least among unmixed height two reduced non arithmetically Cohen-Macaulay curves in $\mathbb{P}^{3}$.

\section{Codimension three Gorenstein algebras}

We now turn to height three Gorenstein ideals. Our approach here is similar to that of the alternative proofs given in the previous section. In [11] the upper bound stated in Conjecture 1.1 was proved for such ideals. The lower bound was proved only when the number of generators is five (or of course three). In this section we will prove an improved version of Conjecture 1.1, and as a consequence we will again (as in the codimension two case) immediately obtain as a corollary that sharpness occurs (necessarily for both bounds) if and only if the resolution is pure.

Let $I \subset R$ be a height three Gorenstein ideal. The possible graded Betti numbers of such ideals were described in [2] and in [3], and it was shown in [6] that any such set of graded Betti numbers in fact occurs for some reduced arithmetically Gorenstein set of points in $\mathbb{P}^{3}$. In fact more was shown in [6].

Remark 3.1. Suppose that $I$ has a minimal graded free resolution

$$
0 \rightarrow R\left(-m_{3}\right) \rightarrow \bigoplus_{i=1}^{2 t+1} R\left(-\beta_{i}\right) \rightarrow \bigoplus_{i=1}^{2 t+1} R\left(-\alpha_{i}\right) \rightarrow I \rightarrow 0
$$

where $\alpha_{1} \leq \cdots \leq \alpha_{2 t+1}, \beta_{1} \leq \cdots \leq \beta_{2 t+1}$ (this is slightly different from the notation of [6]), and $m_{3}=M_{3}$. It was shown in [6] that

- there exists a Cohen-Macaulay ideal $J \subset R$ with minimal graded free resolution

$$
0 \rightarrow \bigoplus_{i=1}^{t} R\left(-\beta_{i}\right) \rightarrow \bigoplus_{i=1}^{t+1} R\left(-\alpha_{i}\right) \rightarrow J \rightarrow 0
$$


- there are homogeneous polynomials $f, g \in J$ of degree $m_{1}(I)=\alpha_{1}$ and $M_{2}(I)=\beta_{2 t+1}=m_{3}-\alpha_{1}$, respectively, such that $\tilde{J}:=(f, g): J$ does not have any components in common with $J$ (i.e. $J$ and $\tilde{J}$ are geometrically linked by $(f, g))$, thus $(f, g)=J \cap \tilde{J}$.

- the ideal $I:=J+\tilde{J}$ has the desired minimal free resolution (6). (Note that not all Gorenstein ideals arise in this way; this only says that numerically for any set of graded Betti numbers this construction produces a Gorenstein ideal with the given Betti numbers, but this is enough for our purposes.)

A famous result of Buchsbaum and Eisenbud in [2] says that without loss of generality we may choose bases so that the middle map of the resolution (6) is represented by a skew symmetric matrix, $M$, and that the minimal generators of $I$ are then given by the maximal Pfaffians of that matrix. However, we may represent the degree matrix $B$ corresponding to $M$, much as we did in (2), so that the entries are increasing from bottom to top and from left to right. Then the resulting degree matrix $B$ is symmetric about the non-main diagonal. In particular, we have

$$
B=\left[\begin{array}{ccccccc}
b_{t} & & & & & & * \\
a_{t} & \ddots & & & & & \\
& \ddots & b_{1} & & & & \\
& & a_{1} & d & & & \\
& & & a_{1} & b_{1} & & \\
* & & & & \ddots & \ddots & \\
* & & & & a_{t} & b_{t}
\end{array}\right]
$$

where

$$
A:=\left[\begin{array}{cccc}
a_{1} & b_{1} & & * \\
& \ddots & \ddots & \\
* & & a_{t} & b_{t}
\end{array}\right]
$$

is the degree matrix of the ideal $J$ (that has been used to produce $I$ ).

Furthermore, comparing the resolutions (6) and (7) we obtain in conjunction with the formulas (3) that

$$
\begin{aligned}
& m_{1}=m_{1}(J)=a_{1}+\ldots+a_{t} \\
& m_{2}=m_{2}(J)=a_{1}+\ldots+a_{t}+b_{t}=m_{1}+b_{t} \\
& m_{3}=d+2\left(b_{1}+\ldots+b_{t}\right) .
\end{aligned}
$$

We now are ready to show our improvement of Conjecture 1.1 for Gorenstein ideals of codimension three.

Proof of Theorem 1.4. Our proof will be by induction on the size of the degree matrix, $A$, of the Buchsbaum-Eisenbud matrix of $I$. First, let $t=1$, i.e. $I$ is a complete intersection. Let $m_{1}, y, M_{1}$ be the degrees of the minimal generators of $I$. Then $m_{1} \leq y \leq M_{1}$ and we get 


$$
\begin{aligned}
& m_{1} m_{2} m_{3}+\left(M_{3}-M_{2}\right)^{2}\left(M_{2}-m_{2}+M_{1}-m_{1}\right) \\
& \quad=m_{1}\left(m_{1}+y\right)\left(m_{1}+y+M_{1}\right)+2 m_{1}^{2}\left(M_{1}-m_{1}\right) \\
& \quad=m_{1}\left(2 y+m_{1}-y\right)\left(3 M_{1}+m_{1}+y-2 M_{1}\right)+2 m_{1}^{2}\left(M_{1}-m_{1}\right) \\
& =6 m_{1} y M_{1}+m_{1}\left[\left(m_{1}+y\right)\left(m_{1}+y-2 M_{1}\right)+\left(m_{1}-y\right) 3 M_{1}+2 m_{1}\left(M_{1}-m_{1}\right)\right] \\
& =6 e(R / I)+m_{1}\left[\left(m_{1}+y\right)\left(y-M_{1}\right)+3\left(m_{1}-y\right) M_{1}+\left(m_{1}-y\right)\left(M_{1}-m_{1}\right)\right] \\
& \leq 6 e(R / I)
\end{aligned}
$$

proving the lower bound.

The upper bound is shown similarly. We have

$$
\begin{aligned}
& M_{1} M_{2} M_{3}-\frac{1}{2} M_{3}\left(M_{2}-m_{2}+M_{1}-m_{1}\right) \\
&=\quad M_{1}\left(y+M_{1}\right)\left(m_{1}+y+M_{1}\right)-\left(m_{1}+y+M_{1}\right)\left(M_{1}-m_{1}\right) \\
&=\quad M_{1}\left(2 y+M_{1}-y\right)\left(3 m_{1}+y+M_{1}-2 m_{1}\right)-\left(m_{1}+y+M_{1}\right)\left(M_{1}-m_{1}\right) \\
&=6 m_{1} y M_{1}+M_{1}\left[\left(M_{1}-y\right)\left(m_{1}+y+M_{1}\right)+2 y\left(y+M_{1}-2 m_{1}\right)\right] \\
&-\left(m_{1}+y+M_{1}\right)\left(M_{1}-m_{1}\right) \\
&= 6 e(R / I)+2 y M_{1}\left(y-m_{1}\right)+\left(m_{1}+y+M_{1}\right) M_{1}\left(M_{1}-y\right) \\
&+\left[2 y M_{1}-\left(m_{1}+y+M_{1}\right)\right]\left(M_{1}-m_{1}\right) \\
& \geq 6 e(R / I)
\end{aligned}
$$

because if $M_{1}=1$ then we must have $m_{1}=y=M_{1}$ and the last estimate becomes an equality. But if $M_{1} \geq 2$ then we get $2 y M_{1}-\left(m_{1}+y+M_{1}\right) \geq$ $2 y M_{1}-\left(2 y+M_{1}\right) \geq 0$ because for any two integers $k, l \geq 2$ we have $k l \geq k+l$. The upper bound follows.

Now assume $t \geq 1$ and let $I^{\prime}$ be the Gorenstein ideals whose degree matrix is

$$
B^{\prime}=\left[\begin{array}{cccccccccc}
b_{t+1} & & & & & & & & \\
a_{t+1} & b_{t} & & & & & & * & \\
& a_{t} & \ddots & & & & & & \\
& & \ddots & b_{1} & & & & & \\
& & & a_{1} & d & & & & \\
& & & & a_{1} & b_{1} & & & \\
& & & & & \ddots & \ddots & & \\
& & & & & & a_{t} & b_{t} & \\
& * & & & & & & a_{t+1} & b_{t+1}
\end{array}\right]
$$


and that has been produced using the Cohen-Macaulay ideal $J^{\prime}$ (cf. Remark 3.1) with degree matrix

$$
A^{\prime}=\left[\begin{array}{ccccc}
a_{1} & b_{1} & & & * \\
& \ddots & \ddots & & \\
& & a_{t} & b_{t} & \\
* & & & a_{t+1} & b_{t+1}
\end{array}\right] .
$$

To simplify notation, we set as in the codimension two case

$$
a:=a_{t+1}, \quad b:=b_{t+1}, \quad \text { and } c:=b_{t} .
$$

Let $I$ be the Gorenstein ideal whose Buchsbaum-Eisenbud matrix is obtained from the Buchsbaum-Eisenbud matrix of $I^{\prime}$ by stripping the top and bottom rows, and the rightmost and leftmost columns such that the degree matrix is $B$ as in (8). Let $m_{1}, m_{2}, m_{3}, M_{1}, M_{2}, M_{3}$ be the invariants of $I$ and let $m_{1}^{\prime}, m_{2}^{\prime}, m_{3}^{\prime}, M_{1}^{\prime}, M_{2}^{\prime}, M_{3}^{\prime}$ be those of $I^{\prime}$. Self-duality of the resolution of $R / I$ provides

$$
M_{1}=m_{3}-m_{2} \quad \text { and } \quad M_{2}=m_{3}-m_{1} .
$$

It follows that

$$
M_{2}-m_{2}=M_{1}-m_{1}=m_{3}-m_{1}-m_{2}=M_{1}+M_{2}-M_{3} .
$$

Thus, the formulas (10) provide

$$
\begin{array}{ll}
m_{1}^{\prime}=m_{1}+a & M_{1}^{\prime}=M_{1}+b+c-a \\
m_{2}^{\prime}=m_{2}+a+b-c & M_{2}^{\prime}=M_{2}+2 b-a \\
m_{3}^{\prime}=m_{3}+2 b & M_{3}^{\prime}=M_{3}+2 b .
\end{array}
$$

We also need the relation between the multiplicities of $R / I$ and $R / I^{\prime}$.

Claim 3.2. $e\left(R / I^{\prime}\right)=e(R / I)+b\left(m_{1}+a\right)\left(M_{2}+b-a\right)$.

To see this, we may assume temporarily that $R / I$ and $R / I^{\prime}$ have dimension one. Thus, the ideals $J$ and $J^{\prime}$ used to produced $I$ and $I^{\prime}$ (as in Remark 3.1) define curves. Denote their arithmetic genera by $g$ and $g^{\prime}$, respectively. As preparation, we first relate the multiplicities of $R / I$ and $R / J$ and then the genera of $J$ and $J^{\prime}$.

Using the notation of Remark 3.1, we have that $I=J+\tilde{J}$ and that $\mathfrak{c}:=J \cap \tilde{J}$ is a complete intersection of type $\left(m_{1}, M_{2}\right)$. Hence, we have graded isomorphisms (cf., e.g., [17], Lemma 3.5)

$$
K_{R / J}\left(4-m_{1}-M_{2}\right) \cong \tilde{J} /(J \cap \tilde{J}) \cong(J+\tilde{J}) / J=I / J
$$

where $K_{R / J}$ denotes the canonical module of $R / J$. It follows that

$$
e(R / I)=\left(m_{1}+M_{2}-4\right) \cdot e(R / J)-(2 g-2) .
$$

Numerically, we may assume that $J^{\prime}$ is a basic double link of $J$ (cf. (4)), i.e. there is a complete intersection $(f, g)$ of type $\left(m_{1}+a, b\right)$ such that $J^{\prime}=f R+g J$. 
Hence using the formula for the genus of a complete intersection (see for instance [15], page 36), Proposition 4.1(b) in [17] provides

$$
g^{\prime}=g+\frac{1}{2} b\left(m_{1}+a\right)\left(m_{1}+a+b-4\right)+b \cdot e(R / J)
$$

Therefore, using formula (14) for $I^{\prime}$ as well as (13) we obtain

$$
\begin{aligned}
e\left(R / I^{\prime}\right)= & \left(m_{1}^{\prime}+M_{2}^{\prime}-4\right) \cdot e\left(R / J^{\prime}\right)-\left(2 g^{\prime}-2\right) \\
= & \left(m_{1}+M_{2}+2 b-4\right)\left[e(R / J)+b\left(m_{1}+a\right)\right] \\
& -\left[2 g+b\left(m_{1}+a\right)\left(m_{1}+a+b-4\right)+2 b \cdot e(R / J)-2\right] \\
= & \left(m_{1}+M_{2}-4\right) \cdot e(R / J)-(2 g-2)+b\left(m_{1}+a\right)\left(M_{2}+b-a\right) \\
= & e(R / I)+b\left(m_{1}+a\right)\left(M_{2}+b-a\right),
\end{aligned}
$$

as claimed.

Now we are ready for the induction step. We assume that the bounds hold for $I$, and we prove them for $I^{\prime}$. We will use the above numbered equations without comment.

We begin with the lower bound. We have to show that

$$
e\left(R / I^{\prime}\right) \geq \frac{1}{6} m_{1}^{\prime} m_{2}^{\prime} m_{3}^{\prime}+\frac{1}{3}\left(m_{1}^{\prime}\right)^{2}\left(m_{3}^{\prime}-m_{1}^{\prime}-m_{2}^{\prime}\right)
$$

Unfortunately, we need some rather lengthy computation. We have

$$
\begin{aligned}
m_{1}^{\prime} m_{2}^{\prime} & m_{3}^{\prime}+2\left(m_{1}^{\prime}\right)^{2}\left(m_{3}^{\prime}-m_{1}^{\prime}-m_{2}^{\prime}\right) \\
= & \left(m_{1}+a\right)\left(m_{2}+a+b-c\right)\left(m_{3}+2 b\right) \\
& +2\left(m_{1}+a\right)^{2}\left(m_{3}-m_{1}-m_{2}-(2 a-b-c)\right) \\
= & m_{1} m_{2} m_{3}+2 m_{1}^{2}\left(m_{3}-m_{1}-m_{2}\right) \\
& +\left[a\left(m_{2}+a+b-c\right)\left(m_{3}+2 b\right)+m_{1}\left(m_{2}+a+b-c\right) 2 b+m_{1}(a+b-c) m_{3}\right] \\
& +2\left[a\left(2 m_{1}+a\right)\left(m_{3}-2 m_{1}-c\right)-\left(m_{1}+a\right)^{2}(2 a-b-c)\right] \\
\leq \quad & 6 e(R / I) \\
& +\left[a\left(m_{1}+a+b\right)\left(m_{3}+2 b\right)+m_{1}\left(m_{1}+a+b\right) 2 b+m_{1}(a+b-c) m_{3}\right] \\
& +2\left[a\left(2 m_{1}+a\right)\left(m_{3}-m_{1}-m_{2}\right)-\left(m_{1}+a\right)^{2}(2 a-b-c)\right]
\end{aligned}
$$

by the induction hypothesis and because of $m_{2}-c=m_{1}$ (by 10). 
Using Claim 3.2 and essentially ordering for $m_{3}$ we obtain

$$
\begin{aligned}
m_{1}^{\prime} m_{2}^{\prime} & m_{3}^{\prime}+2\left(m_{1}^{\prime}\right)^{2}\left(m_{3}^{\prime}-m_{1}^{\prime}-m_{2}^{\prime}\right) \\
\leq \quad & 6 e\left(R / I^{\prime}\right)-6 b\left(m_{1}+a\right)\left(m_{3}-m_{1}+b-a\right) \\
& +\left[a\left(m_{1}+a+b\right)\left(m_{3}+2 b\right)+m_{1}\left(m_{1}+a+b\right) 2 b+m_{1}(a+b-c) m_{3}\right] \\
& +2\left[a\left(2 m_{1}+a\right)\left(m_{3}-m_{1}-m_{2}\right)-\left(m_{1}+a\right)^{2}(2 a-b-c)\right] \\
= & 6 e\left(R / I^{\prime}\right)+m_{3}\left[-6 b\left(m_{1}+a\right)+a\left(m_{1}+a+b\right)+(a+b-c) m_{1}\right] \\
& +2 a\left(2 m_{1}+a\right)\left(m_{3}-2 m_{1}-c\right)-6 b\left(m_{1}+a\right)\left(-m_{1}+b-a\right)+ \\
& a\left(m_{1}+a+b\right) 2 b+m_{1}\left(m_{1}+a+b\right) 2 b-2\left(m_{1}+a\right)^{2}(2 a-b-c) \\
= & 6 e\left(R / I^{\prime}\right)+m_{3}\left[m_{1}(2 a-5 b-c)+a(a-5 b)\right]+ \\
& 2 a\left(2 m_{1}+a\right)\left(m_{3}-2 m_{1}-c\right)-6 b\left(m_{1}+a\right)\left(-m_{1}+b-a\right) \\
& +2 b\left(m_{1}+a\right)\left(m_{1}+a+b\right)-2\left(m_{1}+a\right)^{2}(2 a-b-c) \\
= & 6 e\left(R / I^{\prime}\right)+m_{3}\left[m_{1}(2 a-b-c)+a(a-b)-4 b\left(m_{1}+a\right)\right] \\
& +2 a\left(2 m_{1}+a\right)\left(m_{3}-2 m_{1}-c\right)+2 b\left(m_{1}+a\right)\left(4 m_{1}+4 a-2 b\right) \\
& -2\left(m_{1}+a\right)^{2}(2 a-b-c) \\
= & 6 e\left(R / I^{\prime}\right)+m_{3}\left[m_{1}(2 a-b-c)+a(a-b)\right]+2 a\left(2 m_{1}+a\right)\left(m_{3}-2 m_{1}-c\right) \\
& +4 b\left(m_{1}+a\right)\left(-m_{3}+2 m_{1}+2 a-b\right)-2\left(m_{1}+a\right)^{2}(2 a-b-c) \\
= & 6 e\left(R / I^{\prime}\right)+a(a-b) m_{3}+(2 a-b-c)\left[m_{1} m_{3}+4 b\left(m_{1}+a\right)-2\left(m_{1}+a\right)^{2}\right] \\
& +\left(m_{3}-2 m_{1}-c\right)\left[2 a\left(2 m_{1}+a\right)-4 b\left(m_{1}+a\right)\right]
\end{aligned}
$$

where we used $-m_{3}+2 m_{1}+2 a-b=-m_{3}+2 m_{1}+c+2 a-b-c$. Observing that $a \leq b, a \leq c$, and $m_{3} \geq m_{1}+m_{2}=2 m_{1}+c$ we get

$$
\begin{aligned}
m_{1}^{\prime} & m_{2}^{\prime} m_{3}^{\prime}+2\left(m_{1}^{\prime}\right)^{2}\left(m_{3}^{\prime}-m_{1}^{\prime}-m_{2}^{\prime}\right) \\
\leq & 6 e\left(R / I^{\prime}\right)+(2 a-b-c)\left[m_{1} m_{3}+4 b\left(m_{1}+a\right)-2\left(m_{1}+a\right)^{2}\right] \\
& +\left(m_{3}-2 m_{1}-c\right)\left[4 m_{1}(a-b)+2 a(a-2 b)\right] \\
\leq & 6 e\left(R / I^{\prime}\right)+(2 a-b-c)\left[m_{1} m_{3}+4 b\left(m_{1}+a\right)-2\left(m_{1}+a\right)^{2}\right] \\
\leq & 6 e\left(R / I^{\prime}\right)
\end{aligned}
$$

because

$m_{1} m_{3}+4 b\left(m_{1}+a\right)-2\left(m_{1}+a\right) 2=m_{1}\left(m_{3}-2 m_{1}+4(b-a)\right)+2 a(2 b-a) \geq 0$.

This completes the proof of the lower bound.

Turning to the upper bound, we have to show that:

$$
e\left(R / I^{\prime}\right) \leq \frac{1}{6} M_{1}^{\prime} M_{2}^{\prime} M_{3}^{\prime}-\frac{1}{6} M_{3}^{\prime}\left(M_{1}^{\prime}+M_{2}^{\prime}-M_{3}^{\prime}\right)
$$


To start with, we have:

$$
\begin{aligned}
M_{1}^{\prime} M_{2}^{\prime} & M_{3}^{\prime}-M_{3}^{\prime}\left(M_{1}^{\prime}+M_{2}^{\prime}-M_{3}^{\prime}\right) \\
= & \left(M_{1}+b+c-a\right)\left(M_{2}+2 b-a\right)\left(M_{3}+2 b\right) \\
& -\left(M_{3}+2 b\right)\left(M_{1}+M_{2}-M_{3}+b+c-2 a\right) \\
= & M_{1} M_{2} M_{3}-M_{3}\left(M_{1}+M_{2}-M_{3}\right)+\left(M_{1}+b+c-a\right)(2 b-a)\left(M_{3}+2 b\right)+ \\
& \left(M_{1}+b+c-a\right) M_{2} 2 b+(b+c-a) M_{2} M_{3}-M_{3}(b+c-2 a) \\
& -2 b\left(M_{1}+M_{2}-M_{3}+b+c-2 a\right) \\
\geq & 6 e(R / I)+\left(M_{2}+b-a\right)(2 b-a)\left(M_{3}+2 b\right)+\left(M_{2}+b-a\right) M_{2} 2 b+ \\
& (b+c-a) M_{2} M_{3}-M_{3}(b+c-2 a)-2 b\left(2 M_{2}-M_{3}+b-2 a\right)
\end{aligned}
$$

by the induction hypothesis and because of $M_{2}=M_{1}+c$.

Using Claim 3.2 and essentially ordering for $M_{3}$ we obtain

$$
\begin{aligned}
M_{1}^{\prime} M_{2}^{\prime} & M_{3}^{\prime}-M_{3}^{\prime}\left(M_{1}^{\prime}+M_{2}^{\prime}-M_{3}^{\prime}\right) \\
\geq \quad & 6 e\left(R / I^{\prime}\right)-6 b\left(M_{3}-M_{2}+a\right)\left(M_{2}+b-a\right) \\
& +\left(M_{2}+b-a\right)(2 b-a)\left(M_{3}+2 b\right)+\left(M_{2}+b-a\right) M_{2} 2 b+(b+c-2 a) M_{2} M_{3} \\
& -M_{3}(b+c-2 a)-2 b\left(2 M_{2}-M_{3}+b-2 a\right) \\
= & 6 e\left(R / I^{\prime}\right) \\
& +M_{3}\left[\left(M_{2}+b-a\right)(2 b-a-6 b)+(b+c-a) M_{2}\right] \\
& +\left(M_{2}+b-a\right)\left[(2 b-a) 2 b+2 b M_{2}+6 b\left(M_{2}-a\right)\right] \\
& -M_{3}(b+c-2 a)-2 b\left(2 M_{2}-M_{3}+b-2 a\right) \\
= & 6 e\left(R / I^{\prime}\right) \\
& +M_{3}\left[\left(M_{2}+b-a\right)(-4 b)+(b+c-2 a) M_{2}-a(b-a)\right] \\
& +\left(M_{2}+b-a\right)\left[8 b M_{2}+(2 b-4 a) 2 b\right] \\
& -M_{3}(b+c-2 a)-2 b\left(2 M_{2}-M_{3}+b-2 a\right) \\
= & 6 e\left(R / I^{\prime}\right) \\
& +M_{3}\left[(b+c-2 a) M_{2}-a(b-a)\right] \\
& +\left(M_{2}+b-a\right) 4 b\left[2 M_{2}-M_{3}+b-2 a\right] \\
& -M_{3}(b+c-2 a)-2 b\left(2 M_{2}-M_{3}+b-2 a\right) \\
= & 6 e\left(R / I^{\prime}\right) \\
& +M_{3}\left[(b+c-2 a)\left(M_{2}-1\right)-a(b-a)\right] \\
& +\left(M_{2}+b-a-\frac{1}{2}\right) 4 b\left[2 M_{2}-M_{3}+b-2 a\right]
\end{aligned}
$$

Observe that $b \geq a, c \geq a$, and $M_{2} \geq m_{2} \geq c+1 \geq a+1$. It follows that

$$
\begin{aligned}
b+c-2 a & \geq b-a, \\
M_{2}-1 & \geq a,
\end{aligned}
$$


thus

$$
(b+c-2 a)\left(M_{2}-1\right) \geq a(b-a) .
$$

Since we also have

$$
2 M_{2}-M_{3}+b-2 a=\left(M_{1}+M_{2}-M_{3}\right)+(b+c-2 a) \geq 0
$$

we obtain

$$
M_{1}^{\prime} M_{2}^{\prime} M_{3}^{\prime}-M_{3}^{\prime}\left(M_{1}^{\prime}+M_{2}^{\prime}-M_{3}^{\prime}\right) \geq 6 e\left(R / I^{\prime}\right)
$$

and the upper bound follows.

Remark 3.3. In [20], Srinivasan proved, compared to Conjecture 1.1, stronger bounds for Gorenstein ideals of arbitrary codimension, but with quasi-pure resolutions. A resolution is quasi-pure if $m_{i} \geq M_{i-1}$ for all $i$. In case of a codimension three Gorenstein ideal $I$ her bounds are

$$
\frac{1}{6} m_{1} M_{2} M_{3} \leq e(R / I) \leq \frac{1}{6} M_{1} m_{2} m_{3} .
$$

Note that the lower bound is not even true for arbitrary complete intersections. For example, a complete intersection of type $(2,2,5)$ gives a counterexample. On the other hand, the upper bound is true for all complete intersections and we wonder if it is true for all Gorenstein ideals of codimension three.

The method of proof of Theorem 1.4 also provides an explicit formula for the multiplicity of a Gorenstein ideal in terms of the degrees of the entries of its Buchsbaum-Eisenbud matrix.

Proposition 3.4. Let I be a homogeneous Gorenstein ideal of codimension three with $2 t+1$ minimal generators. Order its Buchsbaum-Eisenbud matrix such that its degree matrix is $B$ as in (8). Then we have

$$
e(R / I)=\sum_{j=1}^{t} b_{j} \cdot\left(a_{1}+\ldots+a_{j}\right) \cdot\left(d+\sum_{i=1}^{j-1}\left(2 b_{i}-a_{i}\right)+b_{j}-a_{j}\right) .
$$

Proof. This follows easily from Claim 3.2. Indeed, let $t=1$. Then $I$ is a complete intersection with minimal generators of degree $a_{1}, b_{1}, d+b_{1}-a_{1}$ and the claim follows. Let $t \geq 2$. Then Claim 3.2 provides the assertion using the formulas (10) and $M_{2}=m_{3}-m_{1}$.

\section{References}

[1] W. Bruns and J. Herzog, Cohen-Macaulay rings. Rev. ed. Cambridge Studies in Advanced Mathematics 39, Cambridge University Press, Cambridge, 1998.

[2] D. Buchsbaum and D. Eisenbud, Algebra structures for finite free resolutions, and some structure theorems for ideals of codimension 3. Amer. J. Math. 99 (1977) 447-485.

[3] S. Diesel, Irreducibility and dimension theorems for families of height 3 Gorenstein algebras. Pacific J. Math. 172 (1996), no. 2, 365-397.

[4] V. Gasharov, T. Hibi and I. Peeva, Resolutions of a-stable ideals. J. Algebra 254 (2002), no. $2,375-394$.

[5] A.V. Geramita and J. Migliore, Hyperplane sections of a smooth curve in $\mathbb{P}^{3}$. Comm. Algebra 17 (1989), no. 12, 3129-3164. 
[6] _ Reduced Gorenstein codimension three subschemes of projective space. Proc. Amer. Math. Soc. 125 (1997), no.4, 943-950.

[7] L.H. Gold, A degree bound for codimension two lattice ideals. J. Pure Appl. Algebra 183 (2003), no.2-3, 201-207.

[8] L.H. Gold, H. Schenck and H. Srinivasan, Betti numbers and degree bounds for some linked zero-schemes. Preprint, 2004.

[9] D.R. Grayson and M.E. Stillman, Macaulay 2, a software system for research in algebraic geometry. Available at http://www.math.uiuc.edu/Macaulay2/.

[10] E. Guardo and A. Van Tuyl, Powers of complete intersections: graded betti numbers and applications. Preprint math. AC/0409090.

[11] J. Herzog and H. Srinivasan, Bounds for multiplicities. Trans. Amer. Math. Soc. 350 (1998), no. 7, 2879-2902.

[12] J. Herzog, N. V. Trung and G. Valla, On hyperplane sections of reduced irreducible varieties of low codimension. J. Math. Kyoto Univ. 34 (1994), no. 1, 47-72.

[13] C. Huneke and M. Miller, A note on the multiplicity of Cohen-Macaulay algebras with pure resolutions. Can. J. Math. 37 (1985), 1149-1162.

[14] J. Kleppe, J. Migliore, R.M. Miró-Roig, U. Nagel and C. Peterson, Gorenstein liaison, complete intersection liaison invariants and unobstructedness. Mem. Amer. Math. Soc. 732 (2001).

[15] J. Migliore, Introduction to liaison theory and deficiency modules. Progress in Mathematics 165, Boston, Birkhäuser, 1998.

[16] J. Migliore, C. Peterson and Y. Pitteloud, Ropes in Projective Space. J. Math. Kyoto Univ. 36 (1996), no. 2, 251-278.

[17] U. Nagel, Even liaison classes generated by Gorenstein linkage. J. Algebra 209 (1998), no. $2,543-584$.

[18] T. Römer, Note on bounds for multiplicities. J. Pure Appl. Algebra 195 (2005), no. 1, 113-123.

[19] T. Sauer, Smoothing projectively Cohen-Macaulay space curves. Math. Ann. 272 (1985), 83-90.

[20] H. Srinivasan, A note on the multiplicities of Gorenstein algebras. J. Algebra 208 (1998), no. $2,425-443$.

Department of Mathematics, University of Notre Dame, Notre Dame, IN 46556

E-mail address: Juan.C.Migliore.1@nd.edu

Department of Mathematics, University of Kentucky, 715 Patterson Office Tower, LeXington, KY 40506-0027

E-mail address: uwenagel@ms.uky.edu

FB Mathematik/Informatik, Universität Osnabrück, 49069 OsnabrüCK, Germany

E-mail address: troemer@mathematik.uni-osnabrueck.de 